\title{
Sinonasal NUT Carcinoma: Delayed Diagnosis Due to the COVID-19 Pandemic and a Review of the Literature
}

\author{
Francesco Maria Crocetta ${ }^{1} \cdot$ Cecilia Botti ${ }^{1,2}$ (D) Martina Fornaciari ${ }^{1}$ - Andrea Castellucci ${ }^{1} \cdot$ Domenico Murri $^{1}$. \\ Giacomo Santandrea $^{2,3} \cdot$ Simonetta Piana $^{3} \cdot$ Angelo Ghidini $^{1}$
}

Received: 10 December 2020 / Accepted: 16 February 2021 / Published online: 8 March 2021

(c) The Author(s), under exclusive licence to Springer Science+Business Media, LLC, part of Springer Nature 2021

\begin{abstract}
NUT carcinoma (NUT-C) is a relatively new malignancy that was recently listed in the 4th edition of the WHO Classification of Head and Neck Tumors in 2017. NUT carcinoma is a rare, aggressive, poorly differentiated carcinoma genetically defined by chromosomal rearrangement of the nuclear protein in testis (NUTMI) gene. The prognosis is extremely poor, with a mean survival $<1$ year. Recent publications suggest a multimodality treatment approach. In the existing literature, only a few reports of sinonasal NUT-C have been reported. Sinonasal NUT-C is considered a very rare entity, but because of its recent inclusion as a head and neck malignancy, its true prevalence is unknown. We report the case of a 56-year-old woman with NUT-C of the sinonasal cavities. In the case reported, the coexistence of Coronavirus disease 2019 (COVID19)-related nasal congestion delayed the diagnosis of NUT-C. Clinical presentation, diagnosis and treatment modalities are discussed together with a review of the literature.
\end{abstract}

Keywords NUT carcinoma $\cdot$ Nose $\cdot$ Paranasal sinuses $\cdot$ Nasal malignancies $\cdot$ COVID-19

\section{Introduction}

NUT carcinoma (NUT-C) is a rare, aggressive, poorly differentiated carcinoma, often with evidence of squamous differentiation, which is genetically defined by chromosomal rearrangement of the nuclear protein in testis (NUTM1) gene [1]. NUT-C harbors a typical somatic $\mathrm{t}(15: 19)$ translocation, where the NUTM1 gene on chromosome 15 is fused to the BRD4 (bromodomain-containing protein 4) gene on chromosome 19 to form the BRD4-NUT fusion oncogene [2, 3]. Less frequently, the $N U T M 1$ gene is fused to a different partner gene, the $B R D 3$ gene or other $N U T$ variant rearrangements [4]. Historically, NUT-C was known as NUT midline carcinoma, as most cases arise in the midline structures of the head, neck and thorax. Currently, it is recognized that

Cecilia Botti

botceci@gmail.com

1 Otolaryngology Unit, Azienda USL-IRCCS di Reggio Emilia, Reggio Emilia, Italy

$2 \mathrm{PhD}$ Program in Clinical and Experimental Medicine, University of Modena and Reggio Emilia, Modena, Italy

3 Pathology Unit, Azienda USL-IRCCS di Reggio Emilia, Reggio Emilia, Italy the nasal cavities and paranasal sinuses are most commonly involved [1].

The prognosis is extremely poor, with a mean survival $<1$ year (6.7 months on average) [5]. There is a paucity of literature regarding the treatment of NUT carcinoma; therefore, NUT-C represents a challenge for providers. Most of the recent publications suggest a multimodality approach combining surgery, radiotherapy and chemotherapy [6].

Herein, we report a case of a 56-year-old woman affected by NUT carcinoma of the nasal cavity and a history of COVID-19 pneumonia. Diagnosis delay and treatment are discussed and compared with previous reports in the literature.

\section{Case Report}

A 56-year-old woman was diagnosed with COVID-19 on the basis of positive nasopharyngeal swabs and radiological findings (i.e., bilateral ground-glass opacities predominately in the lower lobes of the lungs). She had an indolent course characterized by low-grade fever, fatigue, cough, nasal congestion and hyposmia. Nasal congestion persisted after recovery, and she attributed her symptoms to the recent 
infection. She was referred to our otolaryngology clinic because of the appearance of right epiphora, epistaxis and nasal vestibule swelling, in addition to persistent ipsilateral nasal obstruction. Clinical history was negative for wood/ leather dust exposure or tobacco habits. On examination, a violaceous hemorrhagic mass occupied the right nasal fossa, laterally dislocating the right nostril, with negative neck palpation. Fiberendoscopy showed no involvement of the nasopharynx or contralateral nasal cavity. A computed tomography (CT) scan revealed a soft-tissue-density mass occupying the entire right nasal cavity with bone erosion but no cranial base or orbital extension (Fig. 1). On histology, the lesion was composed of sheets of poorly differentiated neoplastic cells with round nuclei and scant cytoplasm (Fig. 2a), which focally showed abrupt areas of keratinization (Fig. 2b, arrow). En masse necrosis was present. Neoplastic cells were immunoreactive with pankeratin (Fig. 2c), p16 and p53. Due to the peculiar morphological appearance, staining with the NUT monoclonal antibody (clone C52B1) was performed with strong nuclear immunoreactivity (Fig. 2 d, inset at higher magnification). A diagnosis of NUT carcinoma was therefore made.

Possible dura or periorbital involvement was ruled out by magnetic resonance imaging (MRI) (Fig. 3a, b). A positron emission tomography (PET)-CT scan ruled out metastatic disease (Fig. 3c). Three weeks after admission, the patient underwent centripetal endoscopic resection. It must be noted that at the time of surgery, the lesion had grown further, causing marked swelling of the nasal vestibule (Fig. 4). The procedure started with debulking of the tumor, followed by right medial maxillectomy, anteroposterior ethmoidectomy, sphenoidotomy and Draf IIa frontal sinusotomy. Careful removal of the entire mucosa near the neoplasm was performed, and surgical margins were carried out. Moreover, the right lamina papyracea was removed to ensure free surgical margins. The suspected site of origin was identified at the insertion of the middle turbinate. The postoperative imaging is reported in Fig. 3d. The postoperative period passed without complications. Bilateral adenomegalies appeared at Robbins level II, and fine-needle aspiration with cytological
Fig. 1 CT scan of nose and paranasal sinuses. a Bone demineralization of ethmoidal cells and the medial maxillary sinus wall in axial section with a bone window. b Soft-tissue-density mass occupying the entire right nasal cavity in axial section with a soft-tissue window. c Bone demineralization of ethmoidal cells, turbinates, and the uncinate process in coronal section with a bone window. d Opacification of the maxillary sinus in coronal section with a soft-tissue window

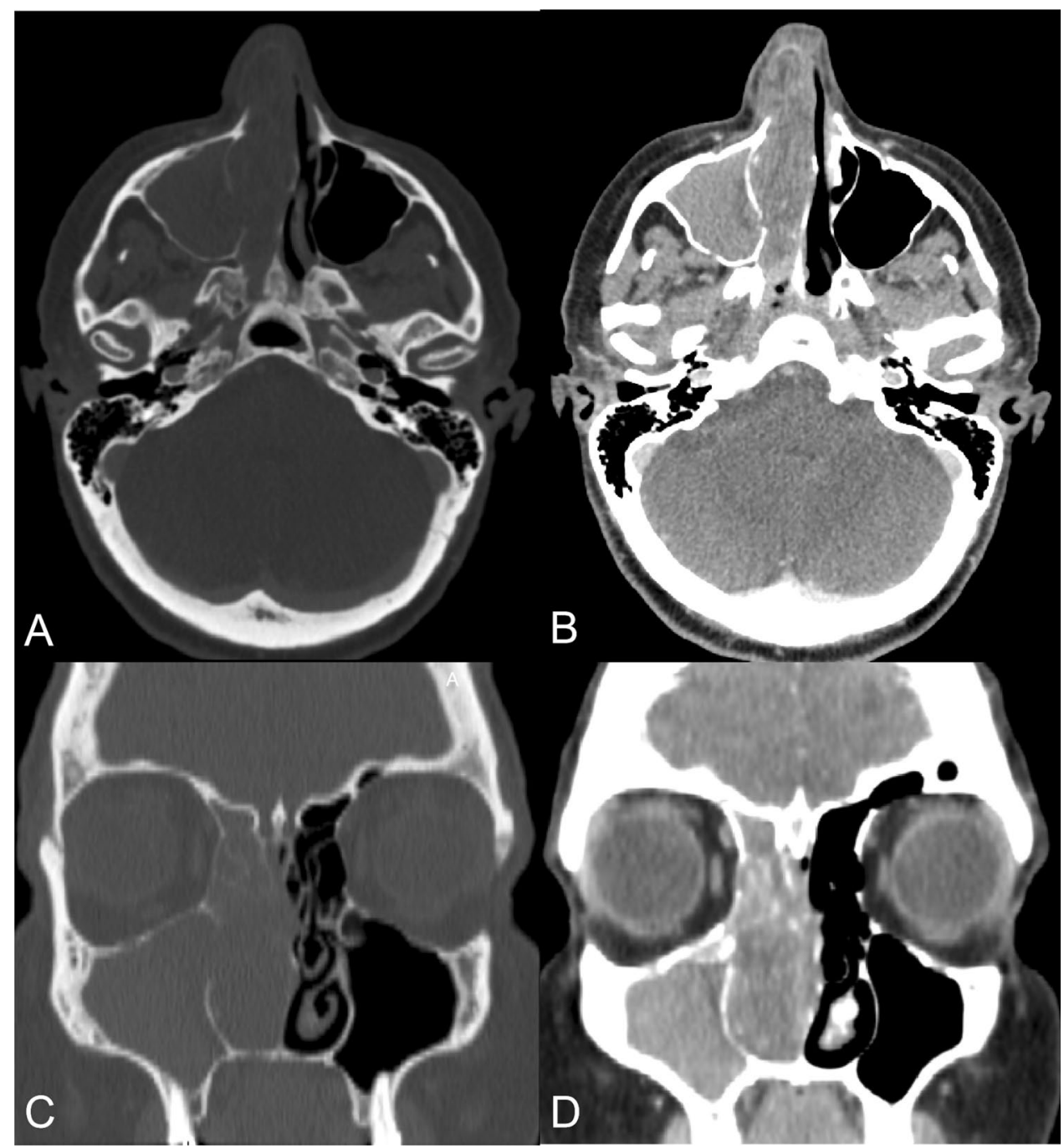




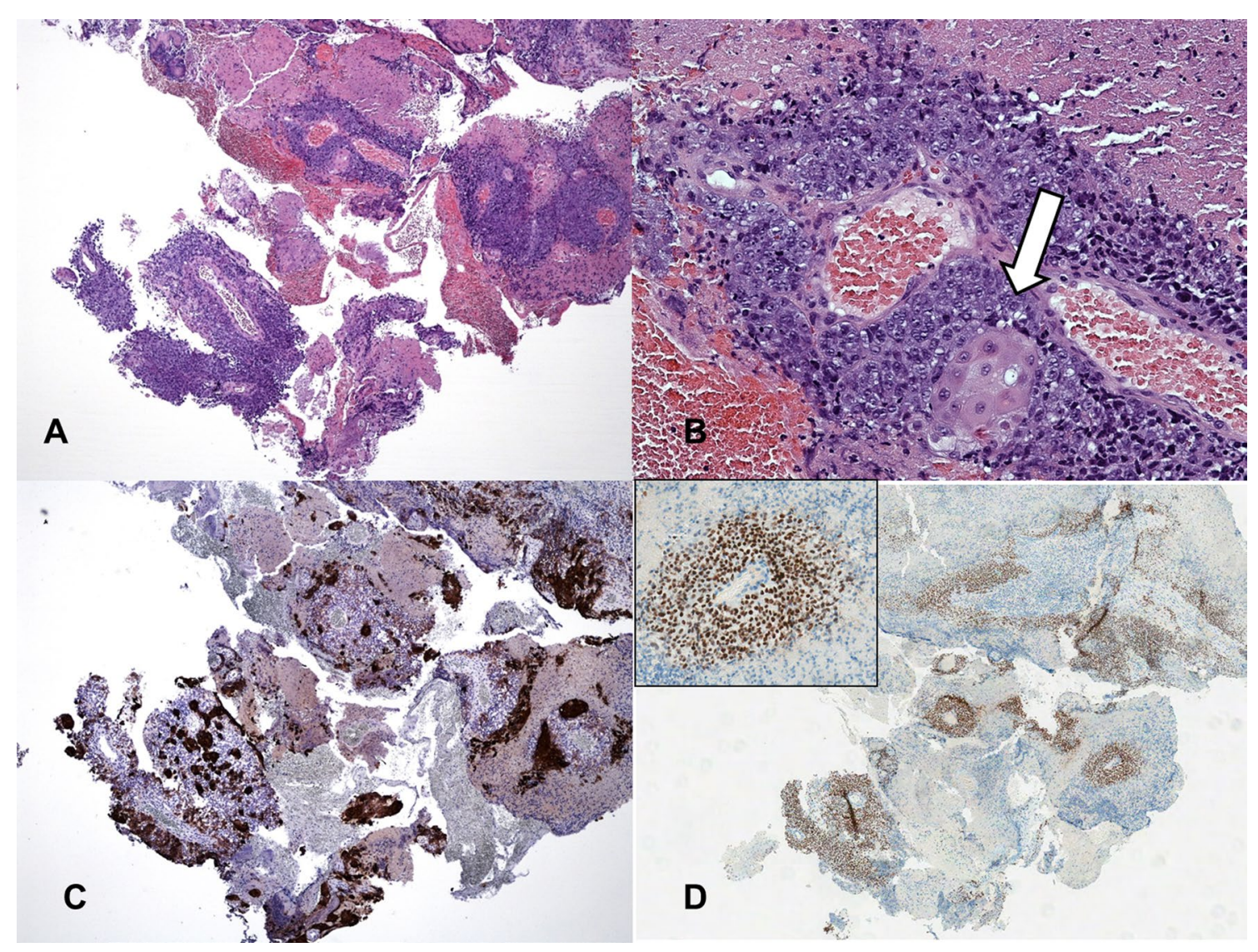

Fig. 2 Histological staining. Poorly differentiated high-grade carcinoma (a) exhibiting focal areas of squamous differentiation (b) within wide areas of necrosis. Immunohistochemical staining was strongly

examination confirmed the metastatic nature on the right side. The patient was promptly administered chemoradiation with cisplatin $\left(100 \mathrm{mg} / \mathrm{m}^{2}\right.$ i.v. every 21 days, 3 cycles $)$ and intensity-modulated radiation therapy (IMRT) with 3 different fields of intensity (60 Gy to the surgical field and right Robbins level II, 59.4 Gy to areas at risk of microscopic disease, 54 Gy to lower risk lateral cervical and retropharyngeal lymph node stations, and additional boosts of $4 \mathrm{~Gy}$ to the surgical field and 6 Gy to the left Robbins level II).

Local recurrence was noted within 1 month. Then, vertebral and liver metastases appeared, and the patient died 6 months later.

\section{Discussion}

\section{Epidemiology}

NUT-C is a relatively new malignancy, as it was recently listed in the 4th edition of the WHO Classification of Head and Neck Tumors in 2017 [1]. In the existing literature, only a few reports of sinonasal NUT-C have been reported. positive with pankeratin (c) and NUT monoclonal antibodies (d inset: positive nuclei at higher magnification)

NUT-C is considered a very rare entity, but the true prevalence in the head and neck is indeed unknown. Recently, Lee et al. [7] reported that NUT-C accounts for $2.9 \%$ and $12.5 \%$ of poorly or undifferentiated carcinomas arising in the head and neck and sinonasal tract, respectively. Furthermore, the authors confirmed that the sinonasal tract represents the most common initial presenting site of the head and neck (73.4\%), followed by the salivary glands (11\%), larynx (7.3\%), pharynx (4.6\%), and oral cavity (3.7\%). People of any age can be affected by a predilection for young adults and a slight female predominance. French [8] described a bimodal peak of incidence in the second and fifth decades. No association with HPV, EBV, other viral infections, smoking or other environmental factors has been described yet [1]. The demographic data of our patient are in line with those described in the literature.

\section{Clinical Course}

NUT-C is characterized by an aggressive clinical course. Most patients present with locally advanced disease at diagnosis. Lymph node or distant metastases may be present in 
Fig. 3 Imaging. a Mass occupying the right sinonasal cavities characterized by predominantly isointense signaling in T2-weighted images. b Heterogeneous contrast enhancement in T1-weighted images; presence of inflammatory stagnation within the right maxillary sinus. c Intense ${ }^{18}$ FDG-PET-CT scan signaling in the right nasal fossa $\left(\mathrm{SUV}_{\max }\right.$ 15.4). d Postsurgical defect in T2-weighted images at MRI

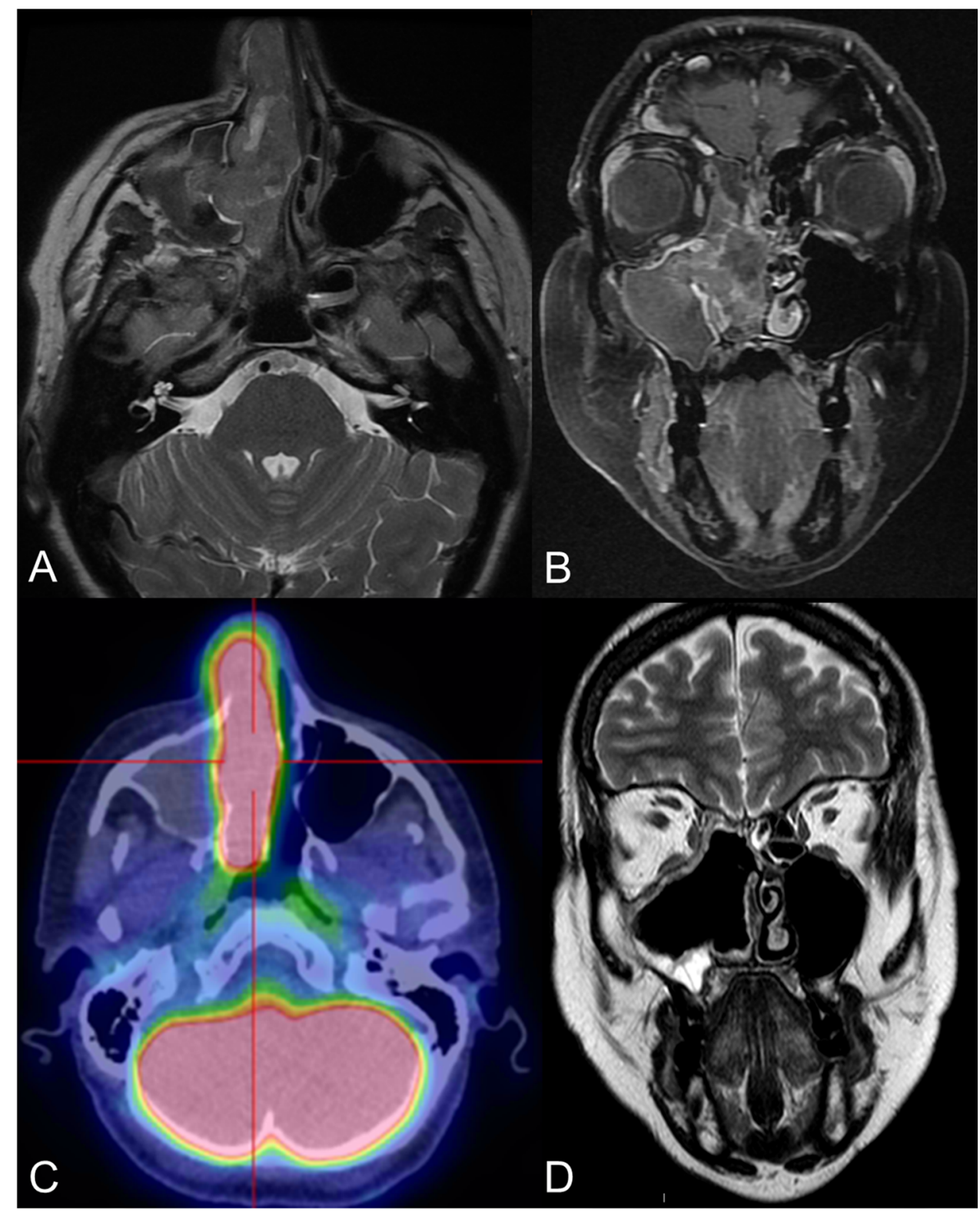

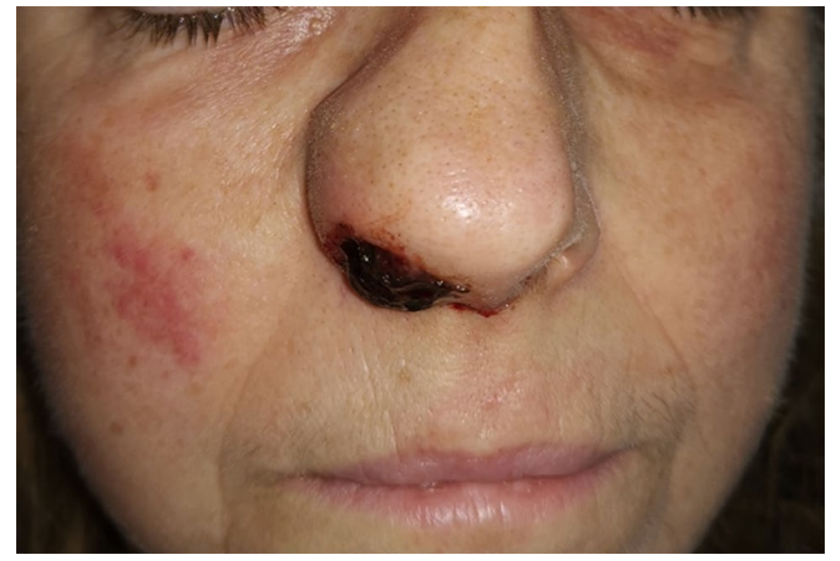

Fig. 4 Nasal vestibule swelling due to the rapid growth of the sinonasal malignancy half of patients at presentation, and if not initially evident, they often develop shortly after diagnosis [9]. As in our patient, the symptoms on presentation are nasal-related (i.e., rhinorrhea, epistaxis, nasal obstruction, facial pain or numbness) and/or eye-related due to local invasion of the orbit (proptosis, diminished vision, periorbital swelling, eyelid edema) or lacrimal sac (epiphora) [10].

\section{Delayed Diagnosis Due to COVID-19}

In the case reported, the coexistence of COVID-19-related nasal congestion delayed the diagnosis of NUT-C, since the patient was referred to an otolaryngologist only after the appearance of further symptoms, in particular nasal swelling and epiphora. This could be explained by two reasons: the 
patients and her family doctor attributed nasal congestion and hyposmia exclusively to COVID-19. Moreover, specialist visits were hardly achievable during the first peak of the pandemic due to the limitation of access to hospitals and clinics because of both the restriction of medical activities and patients' fear of visiting hospitals. These issues concern many medical fields and have been reported in several countries [11-13].

\section{Radiology}

The radiological features of NUT-C are not pathognomonic but are highly suggestive of an aggressive malignant process. NUT-C usually appears as a locally infiltrative/destructive mass, with frequent involvement of the orbit or anterior cranial base. On CT scan, NUT-C has been described as a near-muscle-density mass with faint mineralization within it, without dystrophic calcifications. On MRI, NUT-C has been previously described as a heterogeneous lesion with a predominantly hypointense signal in $\mathrm{T} 1$ and a hyperintense signal in $\mathrm{T} 2$ images, with heterogeneous postcontrast enhancement [14-16].

\section{Diagnosis}

Although possibly suspected in conventional histological slides due to some typical morphological features, the diagnosis of NUT-C requires the demonstration of NUT gene rearrangement by molecular analysis, including fluorescence in situ hybridization (FISH), reverse transcriptase-PCR (RTPCR) or cytogenetic analysis, or by immunohistochemical analysis by using NUT monoclonal antibody C52 to demonstrate the presence of diffuse $(>50 \%)$ nuclear immunoreactivity for NUT. This method has been shown to be $100 \%$ specific and $87 \%$ sensitive for the diagnosis of NUT carcinoma [17]. The introduction of these diagnostic tests could explain the relative increase in the number of diagnosed cases. In fact, before its description, most cases were diagnosed as sinonasal undifferentiated carcinoma (SNUC) or poorly differentiated squamous cell carcinoma [18].

\section{Treatment and Prognosis}

The prognosis of NUT-C is extremely poor, and NUT-C is resistant to conventional treatment modalities. Chau et al. [19] recently published a retrospective survival analysis of all cases of head and neck NUT-C in the International NUT Midline Carcinoma Registry. The authors reported a median overall survival (OS) of 9.7 months (range 6.6-15.6) and a 2-year OS of 30\% (95\% CI 16-46\%) and showed that upfront complete surgical resection with no residuum was associated with significantly improved survival. Conversely, chemotherapy and radiation alone were inadequate, even though both may be considered for adjuvant treatment. Complete surgical excision can be achieved in selected cases of sinonasal NUT-C, but it must be noted that microscopic residuum often persists despite the surgeon's impression of radicality. Therefore, multimodality protocols including aggressive complete surgical resection followed by postoperative radiotherapy and/or chemoradiotherapy are usually advocated. Clinical trials of bromodomain and extraterminal motif (BET) inhibitors [20] and histone deacetylase inhibitors (HDACis) [21] are currently underway, and preliminary results are promising.

Funding This research did not receive any specific grant from funding agencies in the public, commercial, or not-for-profit sectors.

\section{Declarations}

Conflict of interest The authors declare that they have no conflict of interest.

Ethical Approval This research was conducted in accordance with the World Medical Association Declaration of Helsinki (2002). Our Institutional Review Board Area Vasta Emilia Nord does not perform formal ethical assessment for case reports.

Informed Consent Informed consent was obtained from the patient

\section{References}

1. El-Naggar AK, Chan JKC, Grandis JR, Takata T, Slootweg PJ. WHO classification of head and neck tumors. 4th ed. International Agency for Research on Cancer (IARC); 2017.

2. French CA. Demystified molecular pathology of NUT midline carcinomas. J Clin Pathol. 2010;63:492-6.

3. French CA, et al. BRD4-NUT fusion oncogene: a novel mechanism in aggressive carcinoma. Cancer Res. 2003;63(2):304-7.

4. French CA, Rahman S, Walsh EM, et al. NSD3-NUT fusion oncoprotein in NUT midline carcinoma: implications for a novel oncogenic mechanism. Cancer Discov. 2014;4:928-41.

5. Bauer DE, Mitchell CM, Strait KM, et al. Clinicopathologic features and long-term outcomes of NUT midline carcinoma. Clin Cancer Res. 2012;18:5773-9.

6. Arimizu K, Hirano G, Makiyama C, Matsuo M, Sasaguri T, Makiyama A. NUT carcinoma of the nasal cavity that responded to a chemotherapy regimen for Ewing's sarcoma family of tumors: a case report. BMC Cancer. 2018;18(1):1134.

7. Lee T, Cho J, Baek CH, et al. Prevalence of NUT carcinoma in head and neck: analysis of 362 cases with literature review. Head Neck. 2020;42(5):924-38.

8. French CA. Pathogenesis of NUT midline carcinoma. Annu Rev Pathol. 2012. https://doi.org/10.1146/annurev-pathol-01181 $1-132438$.

9. Contrera KJ, Woody NM, Rahman M, Sindwani R, Burkey BB. Clinical management of emerging sinonasal malignancies. Head Neck. 2020. https://doi.org/10.1002/hed.26150. 
10. Napolitano M, Venturelli M, Molinaro E, Toss A. NUT midline carcinoma of the head and neck: current perspectives. Onco Targets Ther. 2019;12:3235-44.

11. Akula SM, Abrams SL, Steelman LS, et al. Cancer therapy and treatments during COVID-19 era. Adv Biol Regul. 2020. https:// doi.org/10.1016/j.jbior.2020.100739.

12. Yang Y, Shen C, Hu C. Effect of COVID-19 epidemic on delay of diagnosis and treatment path for patients with nasopharyngeal carcinoma. Cancer Manag Res. 2020;25(12):3859-64.

13. Harada Y, Shimizu T. Delayed diagnosis of pulmonary thromboembolism due to overfocus on COVID-19. Eur J Case Rep Intern Med. 2020. https://doi.org/10.12890/2020_002002.

14. Dean KE, Shatzkes D, Phillips CD. Imaging review of new and emerging sinonasal tumors and tumor-like entities from the fourth edition of the World Health Organization classification of head and neck tumors. AJNR Am J Neuroradiol. 2019;40(4):584-90.

15. Bair RJ, Chick JF, Chauhan NR, French C, Madan R. Demystifying NUT midline carcinoma: radiologic and pathologic correlations of an aggressive malignancy. AJR Am J Roentgenol. 2014;203(4):391-9.

16. Polsani A, Braithwaite KA, Alazraki AL, Abramowsky C, Shehata BM. NUT midline carcinoma: an imaging case series and review of literature. Pediatr Radiol. 2012;42(2):205-10.
17. Haack H, Johnson LA, Fry CJ, et al. Diagnosis of NUT midline carcinoma using a NUT-specific mono clonal antibody. Am J Surg Pathol. 2009;33:984-91.

18. Franchi A. Epithelial tumors. In: Franchi A, editor. Pathology of sinonasal tumors and tumor-like lesions. Cham: Springer; 2020. https://doi.org/10.1007/978-3-030-29848-7_6.

19. Chau NG, Hurwitz S, Mitchell CM, et al. Intensive treatment and survival outcomes in NUT midline carcinoma of the head and neck. Cancer. 2016;122(23):3632-40.

20. Stathins A, et al. Clinical response of carcinomas harboring the BRD4-NUT oncoprotein to the targeted bromodomain inhibitor OTX015/MK-8628. Cancer Discov. 2016;6(5):492-500.

21. Munster P, Wu N, McMahon M, Gharavi R, Tuck D. Prolonged disease stabilization and tolerability in a nuclear protein in testis midline carcinoma patient treated with dual histone deacetylase and phosphoinositide 3-kinase inhibitor CUDC-907. Case Rep Clin Med. 2018;7:451-60.

Publisher's Note Springer Nature remains neutral with regard to jurisdictional claims in published maps and institutional affiliations. 